\title{
Radiation Calculations for the ILC Cryomodule
}

\author{
N. Nakao, N.V. Mokhov and A. Klebaner \\ Fermi National Accelerator Laboratory \\ P.O. Box 500, Batavia, Illinois 60510
}

April 13, 2007

\begin{abstract}
The MARS 15 radiation simulations were performed for the ILC cryomodule. The model assumes a uniform beam loss intensity of $1 \mathrm{~W} / \mathrm{m}$ of $750-\mathrm{MeV}$ and $250-\mathrm{GeV}$ electron along the inner surface of the beam pipe and the cavity iris of the 12-m cryomodule. Two-dimensional distributions of radiation dose in the module were obtained. Absorbed dose rate and energy spectra of electrons, photons, neutrons and protons were also obtained at the three cryogenic thermometers locations by filling with silicon material in the appropreate locations, and radiation hardness of the thermometers was discussed. From the obtained results, maximum absorbed dose of thermometers at the cooling pipe is $0.85 \mathrm{mGy} / \mathrm{sec}(85$ $\mathrm{mRad} / \mathrm{sec}$ ), that is $0.31 \mathrm{MGy}(31 \mathrm{MRad})$ for 20 years.
\end{abstract}

\section{Introduction}

For the International Linear Collider project (ILC), a superconducting RF cryomodule of the main linac is being designed and its characteristics are tested at Fermilab. Estimation of energy deposition due to electro-magnetic and photo-neutron showers induced by electron beam loss is essential to evaluate the radiation hardness of the electronics devices. Irradiation behaviors of a cryogenic thermometers have been studied for neutrons and photons in Dubna [1,2], and our thermometers should also be tested by experiment and simulation to clarify its characteristics.

Simulations of radiation and energy deposition by the MARS 15 Monte Carlo code [3] were performed with a $250-\mathrm{GeV}$ electron beam for the ILC project, and also with a 750-MeV electron beam for the ILC Test Area facility (ILCTA) planned to be built at Fermilab. From the calculated energy deposition distributions, absorbed dose rates for the cryogenic thermometers at various locations were obtained. Energy spectra of electrons, photons, neturons and protons were also obtained and particle-dependent absorbed doses were clarified and discussed.

\section{Cryomodule Geometry}

A cryomodule geometry for the simulation was made by a simplified design of TESLA at DESY [4]. The main linac consists of many-chain cryomodules to accelerate an electron beam up to $250 \mathrm{GeV}$ in ILC. In this work, simulations in one cryomodule region were carried out.

Figs.1, 2 and 3 show cross-sectional views of the MARS15 cryomodule geometry. One cryomodule structure is about 12-m long and has 8 cavities as shown in Fig.1(a). One cavity has 9 cells (8-9cell cavities) as shown in Fig.1(b). Cavity cells are made with 2.8 -mm-thick niobium and are simplified in a rectangular shape as shown in Fig.1(c). Liquid-helium is flowing between the cavity and 5-mm-thick titanium vessel which is covered with 1-mm-thick mu-metal. 
As shown in Fig.3, the cyromodule itself is covered with a carbon steel casing. A helium gas duct in the center is made of stainless steel. Several cylindrical pipes for a cooling pipe shield are made of aluminum or stainless steel. Alminum thermal shields were simplified to be cylindrical or elliptical shapes.

Silicon material regions of the cryogenic thermometers should be large enough to get good statistics in a Monte Carlo calculation, but this regions should not disturb the radiation field because the actual thermometer size is on a few mm scale. Therefore, silicon material with air density are defined in the rectangular shape inside of the three cylindrical pipes of the cooling pipe shield in the region from 3 to $11 \mathrm{~m}$ in the beam line direction as shown in Fig. 2 to get maximum average absorbed dose.

\section{Beam Loss}

Electrons at a grazing angle of $1 \mathrm{mrad}$ were impacted on the inner radius of the beam pipe uniformly in the circumference and uniformly in entire length of a cryomodule, as shown in Fig. 1(c). The results were normalized to $1 \mathrm{~W} / \mathrm{m}$ beam loss intensity.

Dark current is generated due to an electron field in the wave guide, cavity, RF-gun cathode etc., and is not negligiblly small. However, mechanisms of the dark current generation are complicated, conditiondependent and unclear. In order to simplify the issue in this work, it is assumed that dark current produced from a cathode at RF-gun is dominant and that energy and direction of the dark current electron are equivalent to those of the electron of the primary beam.

\section{Prompt and Absorbed Dose Rate}

A side view of prompt-dose distribution is shown in Fig. 4 for the $250 \mathrm{GeV}$ case. The upper figure shows the entire region of up to $12 \mathrm{~m}$, while the lower figure shows the first $2.8 \mathrm{~m}$. The absolute values were normalized to $1 \mathrm{~W} / \mathrm{m}$ beam loss.

Although, the beam loss is distributed uniformly along $12 \mathrm{~m}$, the promt dose rate profile seems to build up in the upstream region (the first 2-m region) because of secondary-particle forwardness is visible in the simulation with one crymodule. In reality, since several cryomodules are continuously located, dose rate distribution is expected to be almost uniform.

In order to estimate maximum absorbed dose in the thermometers, the region from $3 \mathrm{~m}$ to $11 \mathrm{~m}$ was taken into account (see Fig. 2) so that the maximum average prompt and absorbed dose rates were obtained in the cross sectional view shown in Fig. 5(a) and (b), respectively. Detailed numerical values of the absorbed dose rates at three thermometers regions are discussed in Section 6.

\section{$5 \quad$ Energy Spectra}

Particle energy spectra of photons, electrons, neutrons and protons were scored in the three thermometers regions, and are shown in Fig. 6 for both $750 \mathrm{MeV}$ and $250 \mathrm{GeV}$ electron beams at the thermometer- 2 where the radiation level is the highest of the three. It can be found that spectra shapes and absolute fluences of each particles due to the different beam energies are almost identical in the lower energy region (below $\sim 500 \mathrm{MeV}$ ) when simulation results were normalized to the unit $\mathrm{W} / \mathrm{m}$ beam loss.

Mean energies $\langle E\rangle$ of each spectrum are also shown in Fig. 6. Since fluxes in the low energy region are dominant, the mean energies are all about several $\mathrm{MeV}$ except for protons. 


\section{Relation Between Energy Spectrum and Absorbed Dose}

The real thermometer is about $1-\mathrm{mm}$ thick $\left(0.234 \mathrm{~g} / \mathrm{cm}^{3}\right)$, and $234-\mathrm{cm}$ thick equivalent in air density $\left(10^{3} \mathrm{~g} / \mathrm{cm}^{3}\right)$. In the simulation, the thermometer regions were around 2-cm thickness of air density and much thinner than $234 \mathrm{~cm}$. Therefore, flux-to-absorbed-dose conversion factors for each particle were calculated seperately by MARS15 in a simple geometry with a $234-\mathrm{cm}$ thick silicon using the energy spectra at the thermometer- 2 for the $750 \mathrm{MeV}$ cryomodule in Fig.6. The spectra at the thermometer-1 and 3 were similar and the same conversion factors were assumed. (The difference from those for $250 \mathrm{GeV}$ are negligible, as mentioned in the previous section.) Table 1 gives the obtained conversion factors.

Using the fluxes at three thermister regions in the cryomodule simulations, partial absorbed dose rates by electrons, photons, neutrons and protons for $750 \mathrm{MeV}$ were obtained and are given in Table 2. Although photon flux is highest for all thermometers, absorbed dose by electron is dominant $(60-80 \%)$. The maximum absorbed dose rate is about $0.85 \mathrm{mGy} / \mathrm{sec}(85 \mathrm{mRad} / \mathrm{sec})$ at thermometer-2, and a 20 -year integrated absorbed dose is $0.31 \mathrm{MGy}$ (31 MRad) when 5000-hr annual operation is assumed.

Conversion factors were obtained by the simple geometry calculation also for mono-energetic electrons, photons and neutrons, and fission neutrons whose mean energies are close to those of the spectra at the cryomodule. The values are given also in Table 1. Ratios of these conversion factors to those of the spectra at the cryomodule were obtained, and these ratios are useful for a practical irradiation measurement to investigate the radiation hardness of the thermometer devices prior to the cryomodule operation.

\section{Conclusions}

The MARS 15 simulations were performed for the ILC cryomodule with $750 \mathrm{MeV}$ and $250 \mathrm{GeV}$ electron beam losses. From the simulation results, in the same beam-loss power, the dominant part of energy spectra of particles, prompt dose and absorbed dose are independent of beam energy. The maximum absorbed dose of the cryogenic thermometers at the cooling pipe is $0.85 \mathrm{mGy} / \mathrm{sec}(85 \mathrm{mRad} / \mathrm{sec})$, that is $0.31 \mathrm{MGy}$ (31 $\mathrm{MRad}$ ) for 20 years. Mean energies of electrons, photons and neutrons are around 2-5 MeV, and monoenergetic sources with those energies can be useful for irradiation tests with slight correction factors.

The MARS15 input files created for this study are given in Appendix.

\section{Acknowledgement}

Work supported by Fermi Research Alliance, LLC, under contract No. DE-AC02-07CH11359 with the U.S. Department of Energy.

\section{References}

[1] C.J. Yeager, and S.S. Courts, "A Review of Cryogenic Thermometry and Common Temperature Sensors”, IEEE SENSORS JOURNAL, Vol.1, No.4, December 2001.

[2] Y.P. Filippov, V.V.Golikov, E.N.Kulagin, V.M.Miklayev and A.K.Sukhanova, "In-situ- and Post Irradiation Behaviour of Cryogenic Temperature Sensors Under Fast Neutron Fluence", Advances in Cryogenic Engneering, Vol.47B, p.1700-1707 (2002).

[3] N.V. Mokhov, “The MARS Code System User's Guide”, Fermilab-FN-628 (1995); N.V. Mokhov et al, Fermilab-Conf-04/053 (2004); http://www-ap.fnal.gov/MARS/.

[4] TESLA Technical Design Report-Report, PART-II:TheAccelerator, March (2001) 
Table 1: Flux-to-Absorbed-Dose conversion factors of electrons, photons, neutrons and protons for the energy spectra at the thermometer- 2 in $250 \mathrm{GeV}$ electron beam simulation in Fig. 6 and comparisons with those of various energy spectra.

\begin{tabular}{|c|c|c|c|c|c|}
\hline Particle & Energy spectrum & $\begin{array}{c}\text { Mean } \\
\text { Energy } \\
{[\mathrm{MeV}]}\end{array}$ & $\begin{array}{c}\text { Flux-to-A } \\
\text { conver } \\
{\left[\mathrm{Gy} \mathrm{cm}^{2}\right]}\end{array}$ & $\begin{array}{l}\text { sorbed-Dose } \\
\text { on factor } \\
(\text { Error\%) }\end{array}$ & Ratio \\
\hline \multirow[t]{3}{*}{$\mathrm{e}$} & ILC Cryomodule by $250 \mathrm{GeV}$ e & 11.6 & $2.85 \mathrm{E}-10$ & 0.13 & 1.00 \\
\hline & $5 \mathrm{MeV}$ mono-energy & 5 & $2.77 \mathrm{E}-10$ & 0.08 & 0.97 \\
\hline & $40 \mathrm{MeV}$ mono-energy & 40 & $3.56 \mathrm{E}-10$ & 0.16 & 1.25 \\
\hline \multirow[t]{3}{*}{$\bar{\gamma}$} & ILC Cryomodule by $250 \mathrm{GeV}$ e & 2.6 & $1.65 \mathrm{E}-12$ & 1.72 & 1.00 \\
\hline & Co-60 $\gamma$ & 1.25 & $1.69 \mathrm{E}-12$ & 0.67 & 1.02 \\
\hline & Cs-137 $\gamma$ & 0.664 & $1.84 \mathrm{E}-12$ & 1.8 & 1.12 \\
\hline \multirow[t]{4}{*}{$\mathrm{n}$} & ILC Cryomodule by $250 \mathrm{GeV}$ e & 6.4 & $4.46 \mathrm{E}-12$ & 1.6 & 1.00 \\
\hline & Cf-252 fission neutron & 2.13 & $2.78 \mathrm{E}-12$ & 1.8 & 0.56 \\
\hline & $5 \mathrm{MeV}$ mono-energy & 5 & $6.30 \mathrm{E}-12$ & 1.8 & 1.27 \\
\hline & $2 \mathrm{MeV}$ mono-energy & 2 & $1.32 \mathrm{E}-12$ & 0.90 & 0.27 \\
\hline $\mathrm{p}$ & ILC Cryomodule by $250 \mathrm{GeV}$ e & 207.5 & $1.41 \mathrm{E}-09$ & 0.14 & 1.00 \\
\hline
\end{tabular}

Table 2: Partial and total absorbed dose rates by electrons, photons, neutrons and protons with energy spectra for $250 \mathrm{GeV}$ in three thermometers. Total bsorbed doses for 20 years (5000 hr/yr operation) are also shown. Conversion factors for the energy spectra for the thermometer- 2 were used for all cases.

\begin{tabular}{|c|c|c|c|c|c|c|c|}
\hline & & $\begin{array}{c}\text { Mean } \\
\text { Energy } \\
{[\mathrm{MeV}]}\end{array}$ & $\begin{array}{c}\text { Conversion } \\
\text { Factor } \\
{\left[\mathrm{Gy} \mathrm{cm}^{-2}\right]}\end{array}$ & $\begin{array}{c}\text { Total } \\
\text { Flux } \\
{\left[\mathrm{cm}^{-2} \mathrm{sec}^{-1}\right]}\end{array}$ & $\begin{array}{c}\text { Absorbed } \\
\text { Dose } \\
{\left[\mathrm{Gy} \mathrm{sec}^{-1}\right]}\end{array}$ & & $\begin{array}{l}\text { Ratio } \\
{[\%]}\end{array}$ \\
\hline \multirow[t]{6}{*}{ Thermometer-1 } & $\mathrm{e}$ & 8.6 & $2.85 \mathrm{E}-10$ & $5.34 \mathrm{E}+05$ & $1.52 \mathrm{E}-04$ & & 63.2 \\
\hline & $\gamma$ & 2.2 & $1.65 \mathrm{E}-12$ & $5.35 \mathrm{E}+07$ & $8.82 \mathrm{E}-05$ & & 36.6 \\
\hline & $\mathrm{n}$ & 5.0 & $4.46 \mathrm{E}-12$ & $5.28 \mathrm{E}+04$ & $2.35 \mathrm{E}-07$ & & 0.098 \\
\hline & $\mathrm{p}$ & 214.7 & $1.41 \mathrm{E}-09$ & $2.19 \mathrm{E}+02$ & $3.09 \mathrm{E}-07$ & & 0.12 \\
\hline & & \multirow{2}{*}{\multicolumn{3}{|c|}{$\begin{array}{r}\text { Total Absorbed dose } \\
20 \text {-year }\end{array}$}} & $2.41 \mathrm{E}-04$ & $(0.24 \mathrm{mGy} / \mathrm{sec})$ & \\
\hline & & & & & $8.68 \mathrm{E}+04$ & (0.087 MGy) & \\
\hline \multirow[t]{6}{*}{ "Thermometer-2 } & $\mathrm{e}$ & 11.6 & $2.85 \mathrm{E}-10$ & $2.36 \mathrm{E}+06$ & 6.74E-04 & & 79.1 \\
\hline & $\gamma$ & 2.6 & $1.65 \mathrm{E}-12$ & $1.07 \mathrm{E}+08$ & $1.77 \mathrm{E}-04$ & & 20.7 \\
\hline & $\mathrm{n}$ & 6.4 & $4.46 \mathrm{E}-12$ & $9.88 \mathrm{E}+04$ & 4.41E-07 & & 0.052 \\
\hline & $\mathrm{p}$ & 207.5 & $1.41 \mathrm{E}-09$ & $6.66 \mathrm{E}+02$ & $9.38 \mathrm{E}-07$ & & 0.11 \\
\hline & & \multirow{2}{*}{\multicolumn{3}{|c|}{$\begin{array}{r}\text { Total Absorbed dose } \\
20 \text {-year }\end{array}$}} & $8.52 \mathrm{E}-04$ & $(0.85 \mathrm{mGy} / \mathrm{sec})$ & \\
\hline & & & & & $3.07 \mathrm{E}+05$ & (0.31 MGy) & \\
\hline \multirow[t]{6}{*}{ Thermometer-3 } & $\overline{\mathrm{e}}$ & 9.8 & $2.85 \mathrm{E}-10$ & $5.18 \mathrm{E}+05$ & $1.48 \mathrm{E}-04$ & & 63.0 \\
\hline & $\gamma$ & 2.3 & $1.65 \mathrm{E}-12$ & $5.22 \mathrm{E}+07$ & $8.60 \mathrm{E}-05$ & & 36.7 \\
\hline & $\mathrm{n}$ & 5.1 & $4.46 \mathrm{E}-12$ & $5.24 \mathrm{E}+04$ & 2.34E-07 & & 0.10 \\
\hline & $\mathrm{p}$ & 193.3 & $1.41 \mathrm{E}-09$ & $2.53 \mathrm{E}+02$ & $3.57 \mathrm{E}-07$ & & 0.15 \\
\hline & & \multirow{2}{*}{\multicolumn{3}{|c|}{$\begin{array}{r}\text { Total Absorbed dose } \\
20 \text {-year }\end{array}$}} & $2.34 \mathrm{E}-04$ & $(0.23 \mathrm{mGy} / \mathrm{sec})$ & \\
\hline & & & & & $8.44 \mathrm{E}+04$ & (0.084 MGy) & \\
\hline
\end{tabular}

$* 1 \mathrm{~W} / \mathrm{m}$ beam loss 


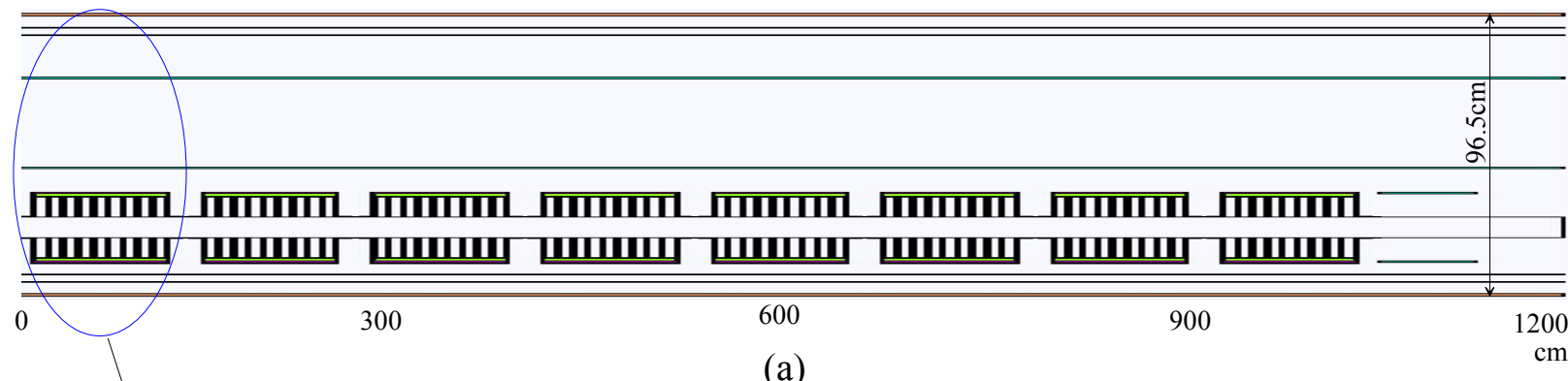

(a)
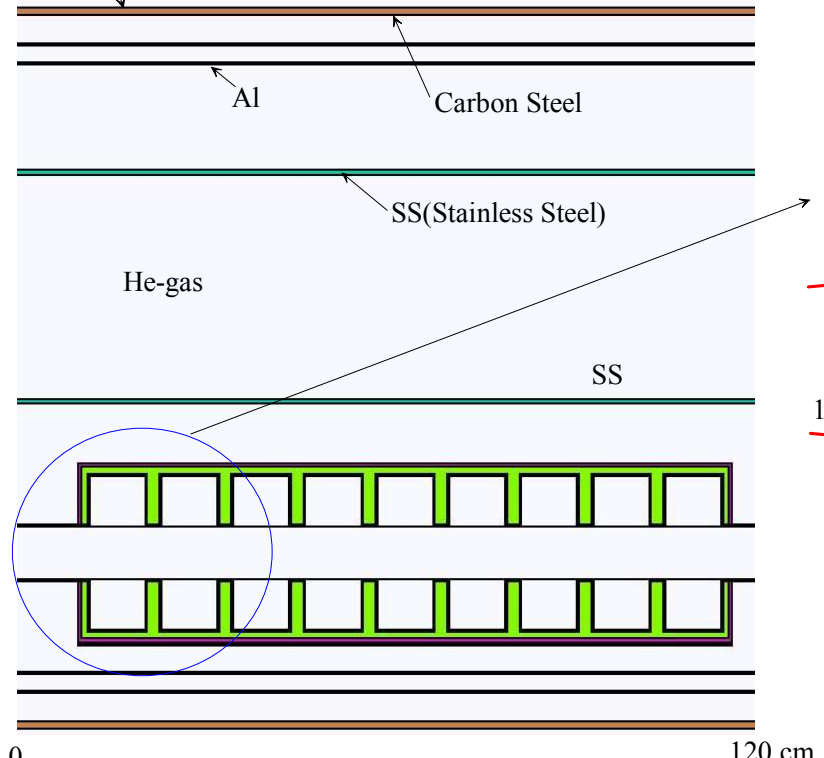

(b)

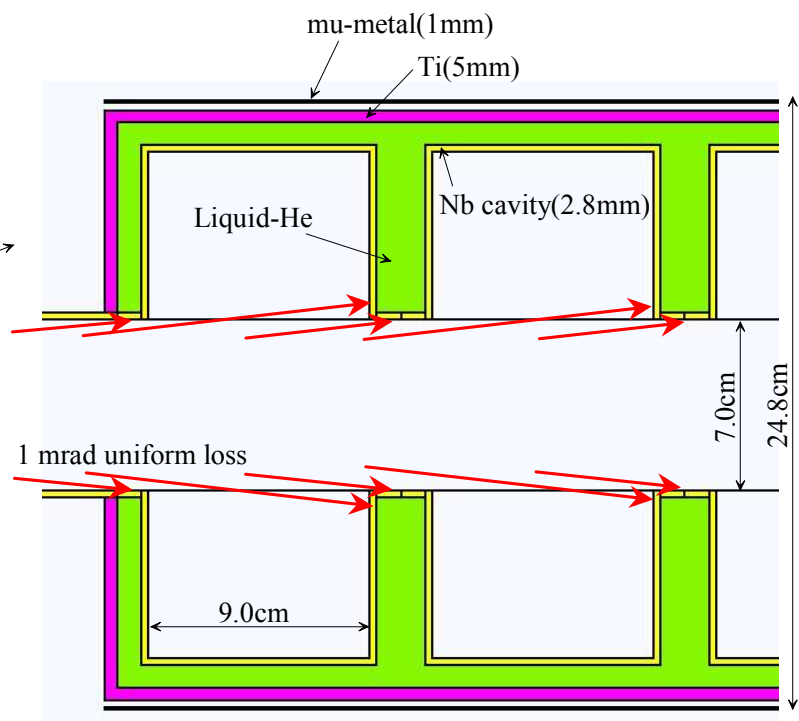

(c)

Figure 1: Side view of cryomodule geometry in vertical plane at A-A' in Fig 3. (a) 12-m whole region, (b) first cavity region and (c) a first few cells of cavity. Sketch of the beam loss image is also shown in (c).

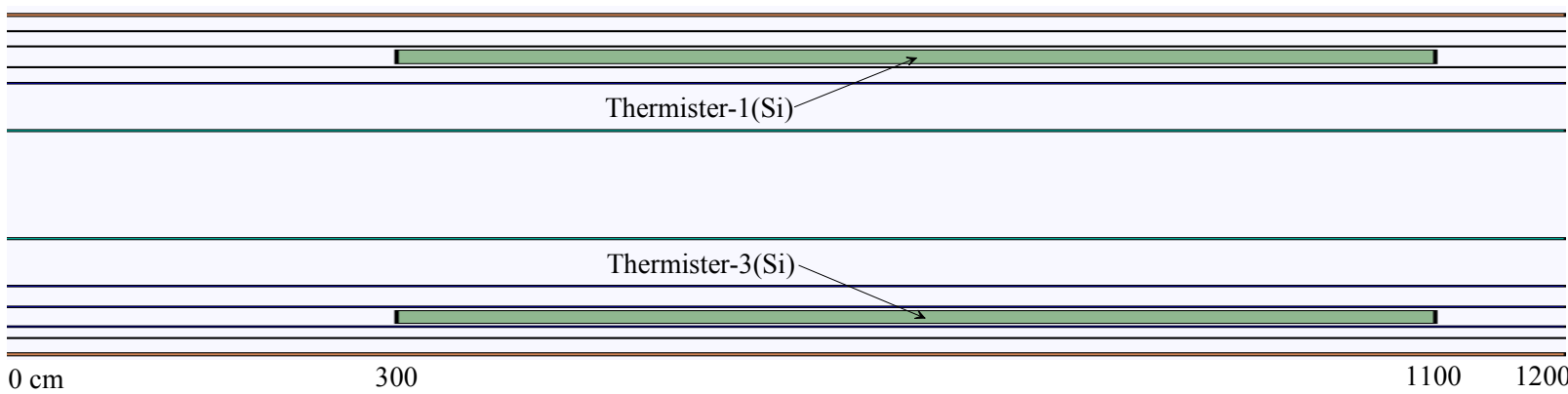

Figure 2: Top view of cryomodule geometry of 12-m whole region in horizontal plane at B-B' in Fig 3. 


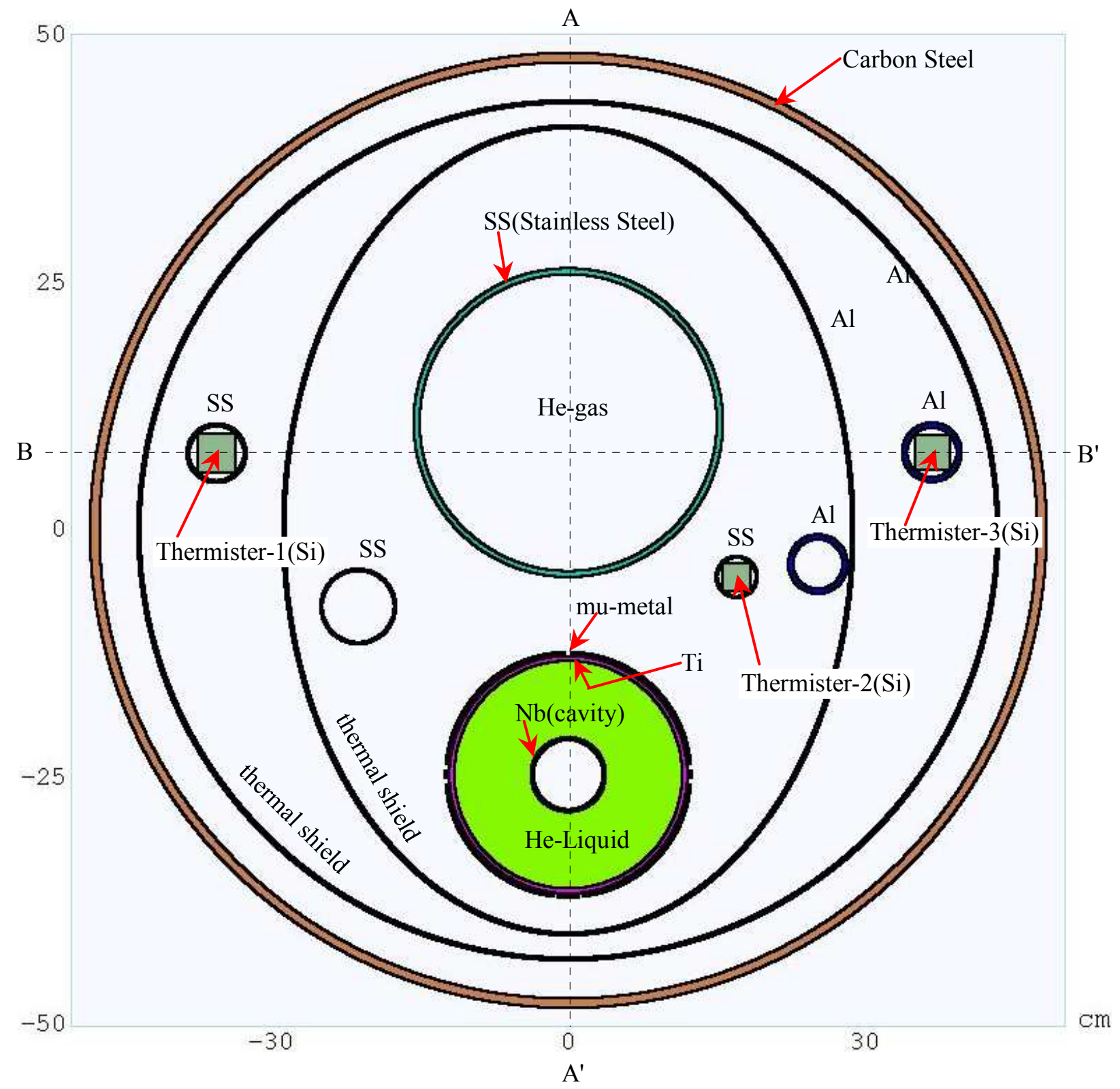

Figure 3: Vertical cross-sectional view of cryomodule geometry at middle part. Vertical A-A' and horizontal B-B' cross-sectional palne views are given in Figs. 1 and 2, respectively. 


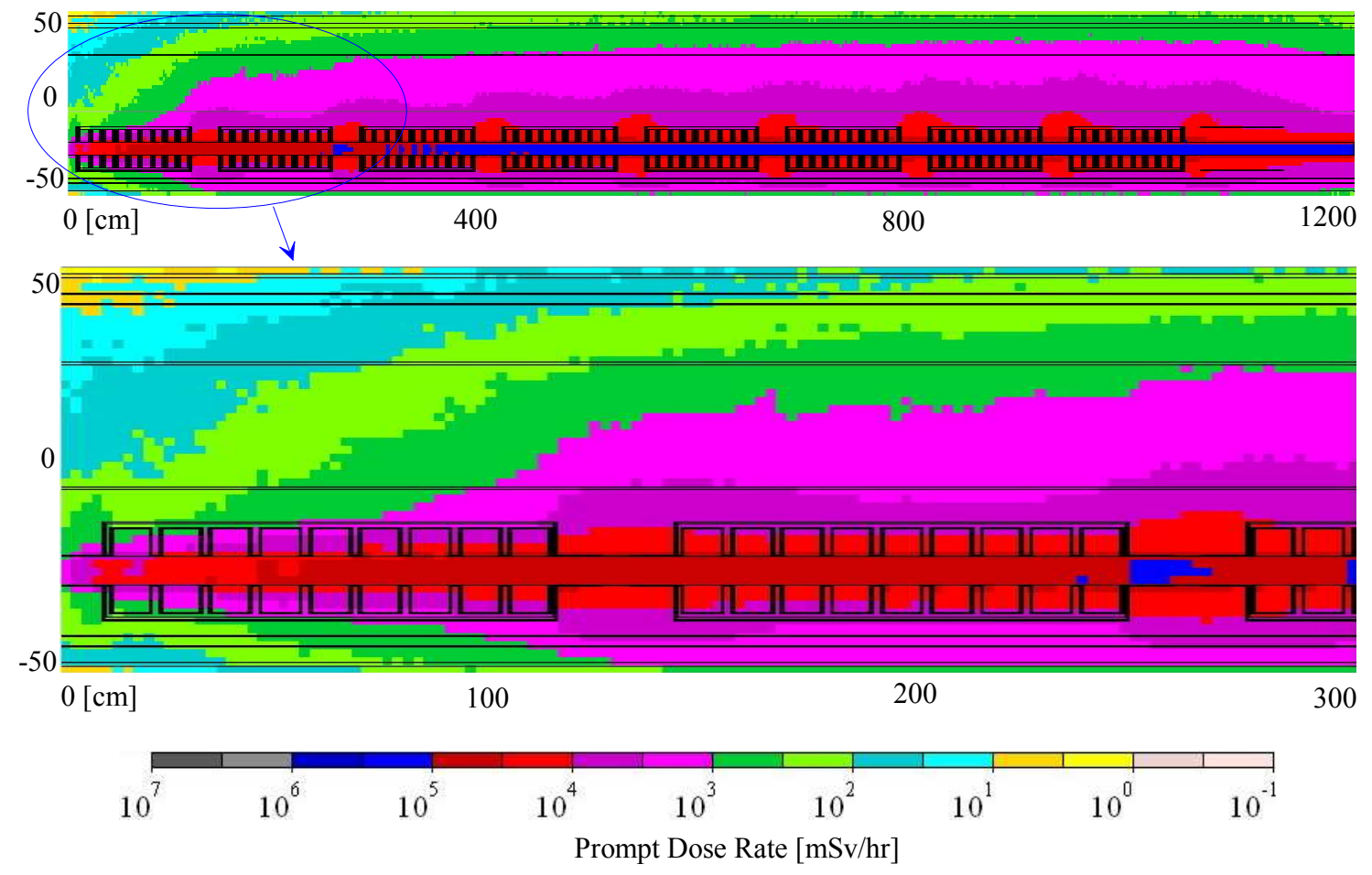

Figure 4: Side view of prompt dose rate distribution in $250 \mathrm{GeV}$ case for the entire region up to 12-m (upper) and the first 2.8-m (lower).

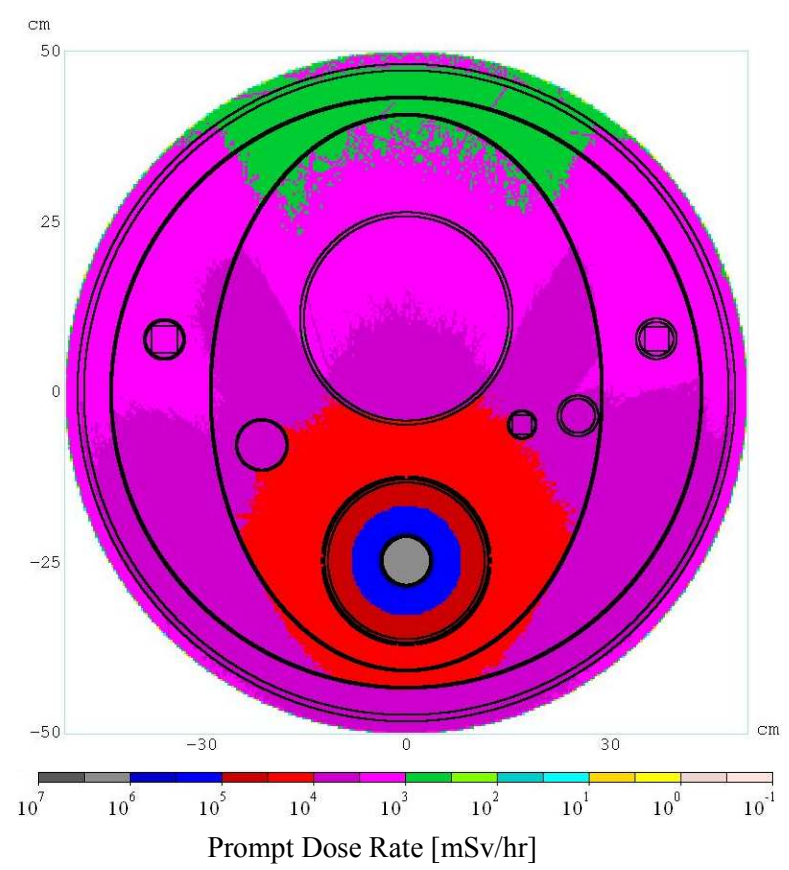

(a)

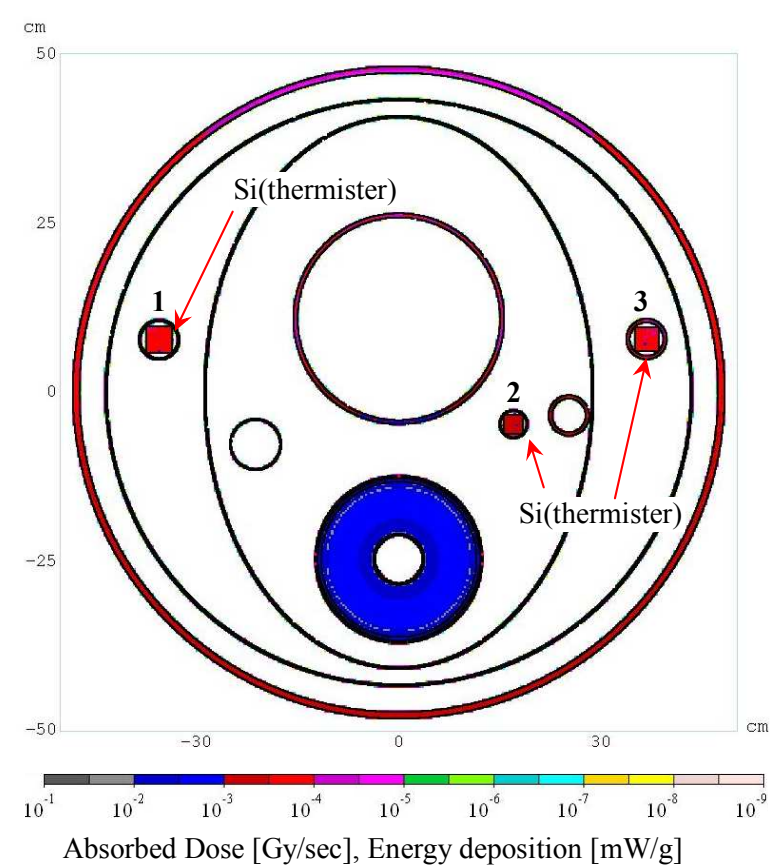

(b)

Figure 5: Cross sectional views of prompt and absorbed dose rates averaged in the region from 3- to 11-m in beam axis. 
Energy Spectra at Thermister-2 of ILC Cryomodule
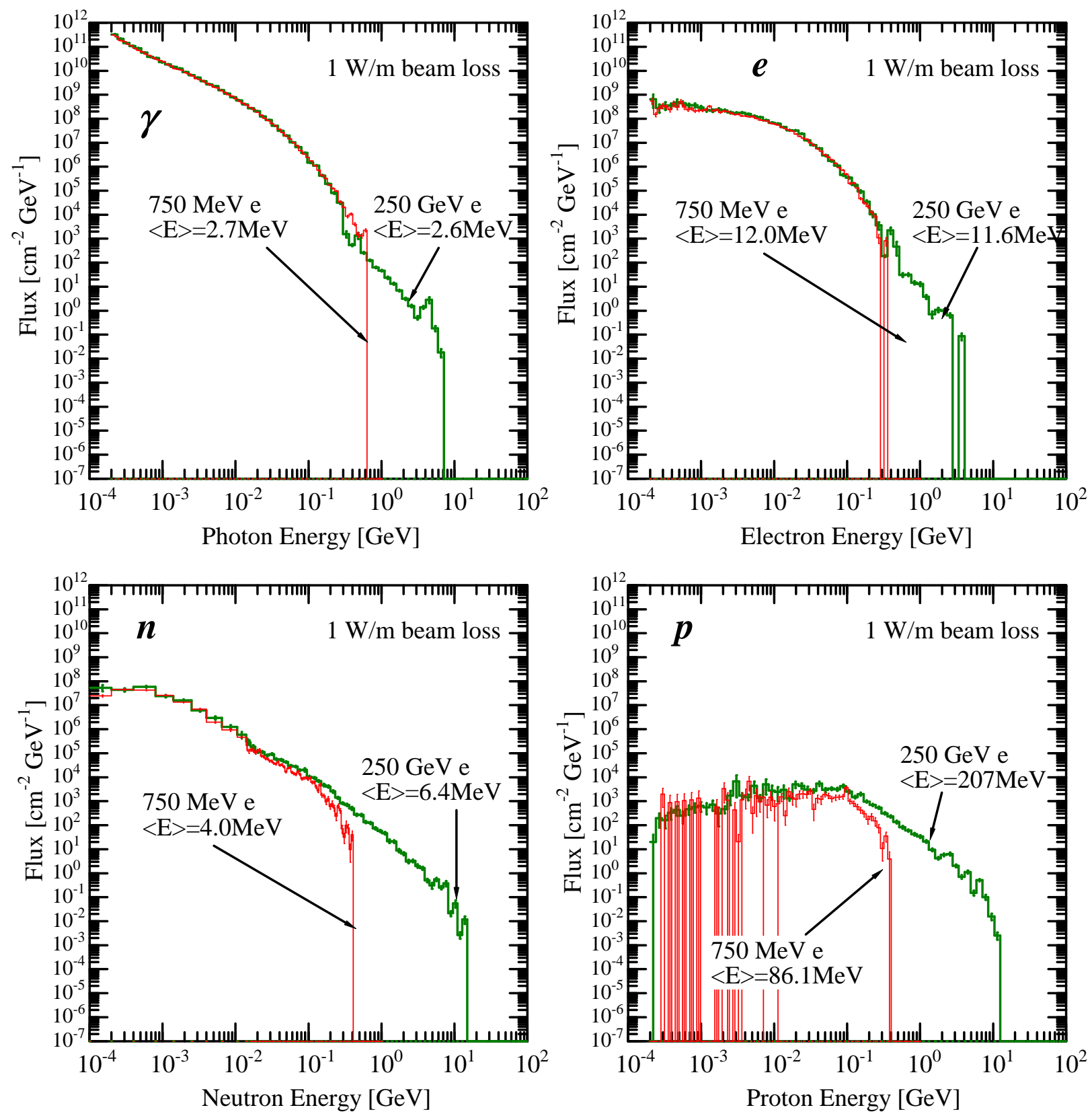

Figure 6: Energy spectra of photons, electrons, neutron and protons at the thermometer-2 location for 1 $\mathrm{W} / \mathrm{m}$ beam loss of $750 \mathrm{MeV}$ and $250 \mathrm{GeV}$ electrons. Mean energies $<E>$ of spectra are also shown in the figure. 


\section{APPENDIX}

\section{MARS.INP}

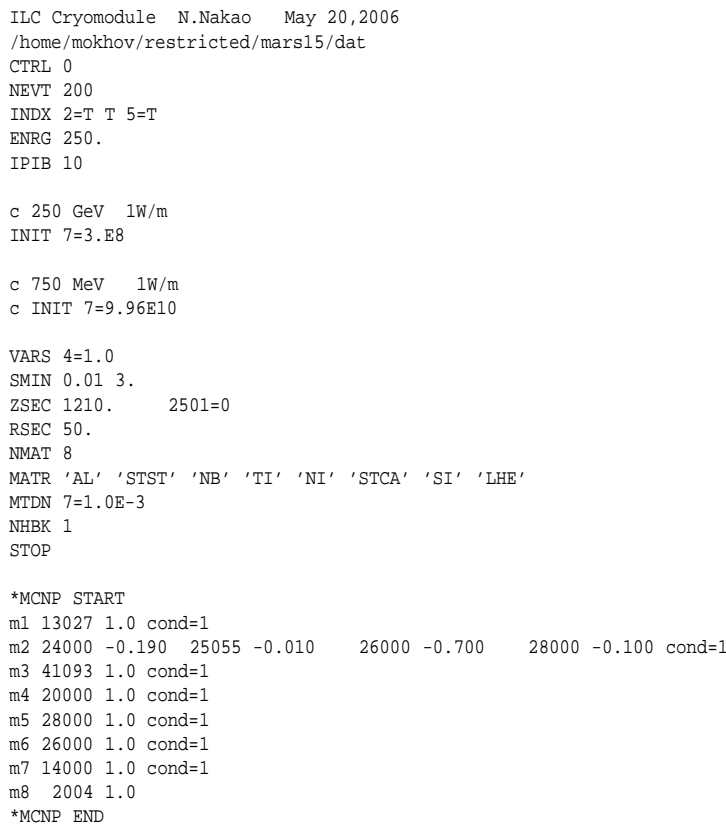

\section{beg1.f}

User subroutine 'beg1'. Beam loss location is uniformly sampled along $\mathrm{r}$ - and $\mathrm{z}$-coordinate. Energy is uniformly sampled in the defined enregy range. Data is read from SRC.DAT

SUBROUTINE BEG1 (JJ, W, E, X, Y, Z, DCX, DCY, DCZ, TOFF , INTA, NREG1) REVISION: $01-D E C-2005$

IMPLICIT DOUBLE PRECISION $(\mathrm{A}-\mathrm{H}, \mathrm{O}-\mathrm{Z})$, INTEGER (I-N) LOGICAL IN

INCLUDE 'azwmat.inc'

INCLUDE ' biount. inc'

TNCLUDE ' bliregl inc'

INCLUDE 'blregl.inc'

INCLUDE 'Cmasnsg. inc'

INCLUDE 'tally2.inc

COMMON/MATINT/IM
$: /$ LOGIND/IND (20)

/BG/EO, ELEAK (3) ,ELGA, ELEN, ELEAMU, ENEUNO, $\operatorname{ALIO}(3), \operatorname{BLEAK}(3,2)$

$: /$ BLTOFF/TOFMIN, TOFMAX, TOFSHF

: /SELEC2/CS, SS, CH, SH

: /HIST/NI, NSTOP, NUPRI, NHIPR

PARAMETER ( $\mathrm{PI}=3.141592653589793227 \mathrm{D}+00)$

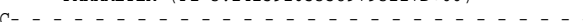

C+++ INSERT YOUR SOURCE TERM HERE +++

SAVE NENTER

DATA NENTER/O/

DATA $z 1, z 2 / 0.0,1200.0$

save zbegin, zend, SRCr, xcavity, alpha, Ene1,Ene2

*** Source term N.Nakao (May 23, 2006)

*** uniform beam loss at SRCr radius from cavity center

*** uniform beam loss along $z$ direction

*** emmition angle is alpha(rad) from $\mathrm{Z}$-coordinate IF (NENTER.EQ.0) THEN

NENTER $=1$

alpha=1.0D-3 ! 1.0mrad angle injection

c alpha $=60 * \mathrm{PI} / 180 . \quad$ ! 60 degree angle injection

open $\left(20\right.$, file $={ }^{\prime}$ SRC. DAT' $)$

read $(20, *)$ xcavity

read $(20, *)$ zbegin, zend $X$ or cavitycenter

$\operatorname{read}(20, *) \mathrm{SRCr}$ ! source location in radius

write $\left({ }^{*},{ }^{*}\right)$ xcavity, ${ }^{\prime}$ ! X of cavitycenter

write $\left({ }^{*}, *\right)$ zbegin, zend,' ! zbegin, zend'

write $\left({ }^{*},{ }^{*}\right) \mathrm{SRCr}^{\prime}, \quad$ ! source location in radius

read $(20, *)$ Ene1 ! Kinetic Energy of entrance of cryomodule

read $(20, *)$ Ene2 ! Kinetic Energy of exit of cryomodule

close $(20)$

write $(*, *)$ Ene1, ! Kinetic Energy of entrance of cryomodule

write $(*, *)$ Ene2,' ! Kinetic Energy of exit of cryomodule'
ENDIF

theta $=2.0 \mathrm{~d} 0 * \mathrm{pi} *$ rand ()

$\mathrm{z}=($ zend-zbegin $)$ *rand ()$+$ zbegin

$\mathrm{x}=\mathrm{SRCr}{ }^{*} \sin ($ theta $)+\mathrm{xcavit}$

$\mathrm{y}=\mathrm{SRCr}{ }^{*} \operatorname{Cos}($ theta $)$

dcx $=\sin ($ theta $) * \sin ($ alpha

dcy $=\cos ($ theta) $* \sin ($ alpha)

$d c z=\operatorname{sqrt}\left(1-d c c^{\star *} 2-d c y^{\star *} 2\right.$

$E=(E n e 2-E n e 1) /(z 2-z 1) *(z-z 1)+$ Ene1 ! electron energy

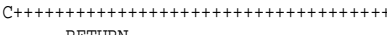

\section{SRC.DAT}

Data file of beam loss location and energy range for beg1 subroutine.

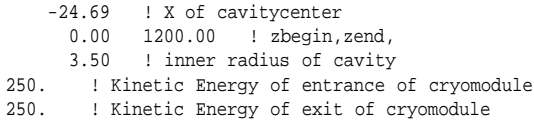

250. ! Kinetic Energy of exit of cryomodule 


\section{geom.f}

Fortran program to make GEOM.INP easily.

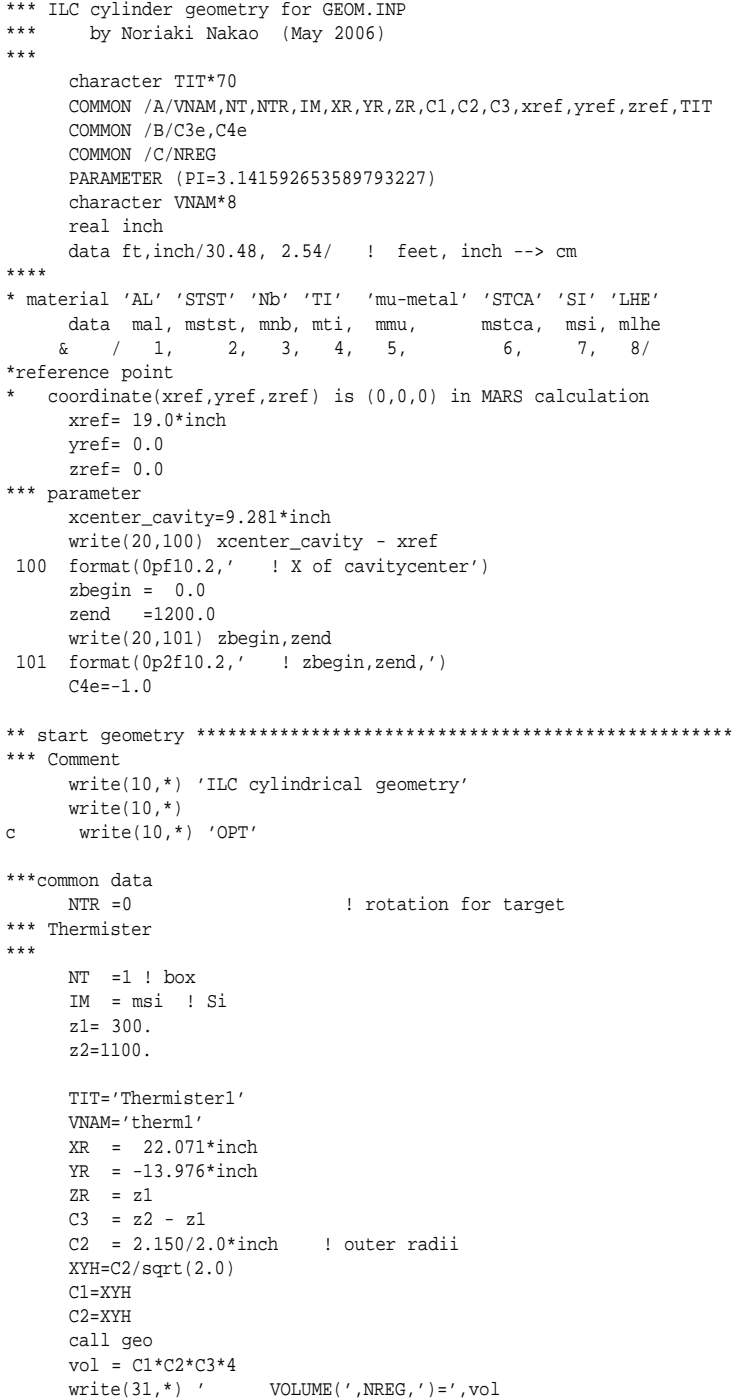

101 format (0p2f10.2, ! zbegin, zend,') $\mathrm{C} 4 \mathrm{e}=-1.0$

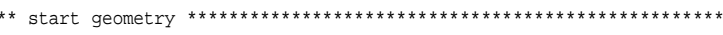

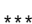

TIT $=^{\prime}$ Cylinder outside VNAM $=$ ' Cyl-out'

NT $=2 \quad$ ! cylinder

TM = mstca $\mid$ carbon steel

$\mathrm{XR}=19.0 *$ inch

$\mathrm{YR}=0.0$

$\mathrm{C} 1=37.25 / 2.0 *$ inch $!$ inner radii

$\mathrm{C} 2=38.00 / 2.0 *$ inch ! outer radii

call geo

TIT $={ }^{\prime}$ Cylinder inside'

VNAM $=^{\prime}$ Cyl-in'

NT $=2$

$\mathrm{IM}=\operatorname{mal} \quad ! \mathrm{al}$

$\mathrm{C} 1=34.00 / 2.0 *$ inch $!$ inner radi

$\mathrm{C} 2=\mathrm{C} 1+0.3$ ! outer radii

TIT='Elliptical Tube' VNAM $=$ ' Elliptic'

NT $=6$

! Elliptical Tube

$\mathrm{IM}=\mathrm{mal} \quad ! \mathrm{Al}$

$\mathrm{C} 1=32 \cdot 0 / 2 \cdot 0 \star$ inch

$\mathrm{C} 2=22.5 / 2.0 *$ inch

$\mathrm{C} 3 \mathrm{e}=0.3$

$\mathrm{C} 4 \mathrm{e}=$ zend

! inner radii of $x$-zxis

! inner radii of $\mathrm{y}$-zxis

! tube wall thickness

call geo

! tube z-length

***

TIT $=^{\prime}$ Cylinder center

VNAM $=^{\prime}$ Cyl-cnt'

NT $=2$

cylinder

$\mathrm{IM}=$ mstst $\quad !$ STST stainles steel

$\mathrm{XR}=23.291 *$ inch

$\mathrm{YR}=0.0$

$\mathrm{C} 1=11.811 / 2.0 *$ inch $!$ inner radi

$\mathrm{C} 2=12.283 / 2.0 *$ inch $\quad$ ! outer radii

call geo

TIT $=$ ' pipe 1 for thermister $-1^{\prime}$

VNAM $=^{\prime}$ Cyl $-p 1^{\prime}$

NT $=2$ ! cylinder

IM = mstst $\quad !$ STST stainles steel

$\mathrm{XR}=22.071 *$ inch

$\mathrm{YR}=-13.976 *$ inch

$\mathrm{C} 1=2.156 / 2.0 *$ inch $\quad !$ inner radii

C2 $=2.374 / 2.0 *$ inch $\quad$ ! outer radii

call geo

TIT $=$ 'pipe'

$\mathrm{VNAM}={ }^{\prime}$ Cyl $-\mathrm{p}^{\prime}$

(T)

IM $=$ mstst ! STST stainles steel

$\mathrm{XR}=15.974 *$ inch
$\mathrm{YR}=-8.347 *$ inch

$\mathrm{C} 1=2.839 / 2.0 *$ inch $\quad$ ! inner radii

$\mathrm{C} 2=2.996 / 2.0 *$ inch $\quad$ ! outer radii

call geo

TIT=' pipe2 for thermister $-2^{\prime}$

VNAM $={ }^{\prime}$ Cyl $-\mathrm{p}^{\prime}$

NT $=2 \quad$ ! cylinder

IM = mstst ! STST stainles stee

$X R=17.150 *$ inch

$\mathrm{YR}=6.693 *$ inch

$\mathrm{C} 1=1.531 / 2.0 *$ inch $\quad$ ! inner radii

$\mathrm{C} 2=1.661 / 2.0 *$ inch ! outer radii

$$
\text { call geo }
$$

TIT $={ }^{\prime}$ Al pipe for thermister -3 VNAM $=$ ' ALPip $3^{\prime}$

NT $=2 \quad$ ! cylinder

IM = mal $!$ AL aluminum

$\mathrm{XR}=17.662 *$ inch

$\mathrm{YR}=9.923 *$ inch

C1 $=1.968 / 2.0 *$ inch $!$ inner radi

C2 $=2.362 / 2.0 *$ inch ! outer radii

$\star \star \star$ call geo

TIT='Al pipe

VNAM $=^{\prime}$ ALPip

NT $=2$

! cylinder

IM $=$ mal $\quad$ AL aluminum

$\mathrm{XR}=22.110 *$ inch

$\mathrm{YR}=14.449 *$ inch

$\mathrm{C} 1=1.969 / 2.0 *$ inch $\quad$ ! inner radii

C2 $=2.362 / 2.0 *$ inch ! outer radi

call geo

$* * *$ cavity $* * *$ 


\section{GEOM.INP}

ILC cylindrical geometry

! (NREG $=2, \mathrm{NEXG}=1$ ) Thermiste

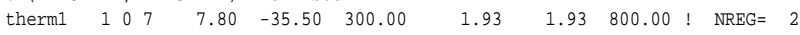

! (NREG $=3$, NEXG $=2$ ) Thermister2

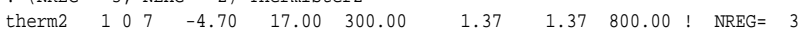

! $\quad$ (NREG $=4$, NEXG $=3$ ) Thermister 3

therm3 $\begin{array}{llllll}1 & 0 & 7 & 7.90 & 36.70 & 300.00\end{array}$

! (NREG $=5$, NEXG $=4$ ) Cylinder outside

Cyl-out $\begin{array}{llllll}2 & 0 & 6 & 0.00 & 0.00 & 0.00\end{array}$

! (NREG $=6$, NEXG $=5)$ Cylinder inside

Cyl-in $\begin{array}{llllll}2 & 0 & 1 & 0.00 & 0.00 & 0.00\end{array}$

! (NREG $=7$, NEXG $=6$ 6) Elliptical Tube

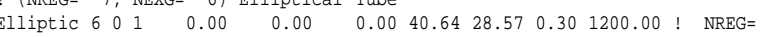

! (NREG $=8$, NEXG $=7$ ) Cylinder center

Cyl-cnt $\begin{array}{llllllllll}2 & 0 & 2 & 10.90 & 0.00 & 0.00 & 15.00 & 15.60 & 1200.00\end{array} \quad$ NREG $=8$

! (NREG $=9$, NEXG $=8$ ) pipe1 for thermister -1

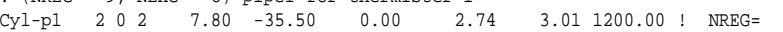

! (NREG $=10$, NEXG $=9$ ) pip

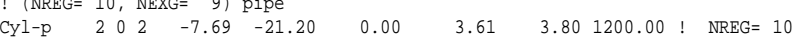

! (NREG $=11$, NEXG $=10)$ pipe2 for thermister -2

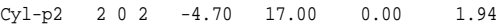

$2.111200 .00 \quad ! \quad \mathrm{NREG}=11$

! (NREG= 12, NEXG $=11$ ) Al pipe for thermister -3

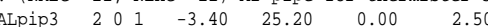

! (NREG $=13$, NEXG $=12)$ Al pipe

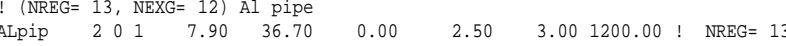

! (NREG $=14$, NEXG $=13$ ) Vacuum inside the cavity and HOM

Vacuum $\begin{array}{lllllllll}2 & 0 & 0 & -24.69 & 0.00 & 0.00 & 0.00 & 3.50 & 1200.00\end{array}$ ～NREG $=14$

! $\quad$ NREG $=15$, NEXG $=14)$ HOM coupler

HOM $\quad \begin{array}{lllllllll}2 & 0 & 3 & -24.69 & 0.00 & 0.00 & 3.50 & 3.78 & 10.56\end{array} ! \quad$ NREG $=15$

! (NREG= 16, NEXG $=15)$ Nb cavity inside narrow Iris

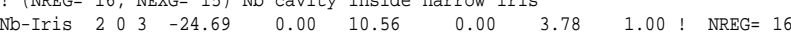

! (NREG= 17, NEXG $=16)$ Vacuum inside the cavity

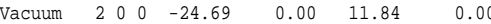

$10.30 \quad 8.98 \quad ! \quad \mathrm{NREG}=17$

! $(\mathrm{NREG}=18, \mathrm{NEXG}=17) \mathrm{Nb}$ cavity inside wide

$\begin{array}{llllllllll}\mathrm{Nb}-\text {-cavi2 } & 2 & 0 & 3 & -24.69 & 0.00 & 11.56 & 0.00 & 10.58 & 9.54\end{array} \quad$ NREG $=1$

! (NREG $=19$, NEXG $=18$ ) Nb cavity inside narrow Iris

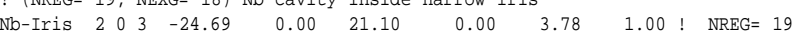

! (NREG $=20$, NEXG $=19) \quad \mathrm{Nb}$ cavity inside narrow Iris

$\begin{array}{llllllllll}\mathrm{Nb} \text {-Iris } & 2 & 0 & 3 & -24.69 & 0.00 & 22.10 & 0.00 & 3.78 & 1.00\end{array} ! \mathrm{NREG}=20$

! (NREG $=21$, NEXG $=20$ ) Vacuum inside the cavity

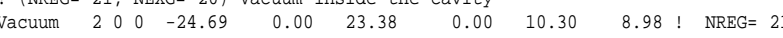

! (NREG= 22, NEXG $=21) \mathrm{Nb}$ cavity inside wide

$\begin{array}{lllllllllll}\mathrm{Nb}-\text { cavi2 } & 2 & 0 & 3 & -24.69 & 0.00 & 23.10 & 0.00 & 10.58 & 9.54 & !\end{array}$

! (NREG $=23$, NEXG $=22) \mathrm{Nb}$ cavity inside narrow Iris

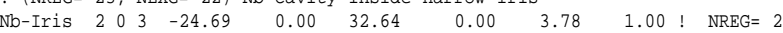

! (NREG $=24, \mathrm{NEXG}=23$ ) $\mathrm{Nb}$ cavity inside narrow Iris

Nb-Iris $\begin{array}{llllllll}2 & 0 & 3 & -24.69 & 0.00 & 33.64 & 0.00 & 3.78\end{array}$

! (NREG $=25$, NEXG $=24$ ) Vacuum inside the cavity

$\begin{array}{llllllllll}\text { Vacuum } & 2 & 0 & 0 & -24.69 & 0.00 & 34.92 & 0.00 & 10.30 & 8.98\end{array} \quad$ NREG $=25$

! (NREG $=26, \quad$ NEXG $=25) \mathrm{Nb}$ cavity inside wide

$\begin{array}{llllllllll}\mathrm{Nb} \text {-cavi2 } & 2 & 0 & 3 & -24.69 & 0.00 & 34.64 & 0.00 & 10.58 & 9.54\end{array} \quad \mathrm{NREG}=26$

! (NREG $=27, \mathrm{NEXG}=26) \mathrm{Nb}$ cavity inside narrow Iris

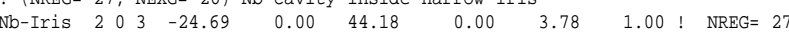

! (NREG $=28, \mathrm{NEXG}=27$ ) $\mathrm{Nb}$ cavity inside narrow Iris

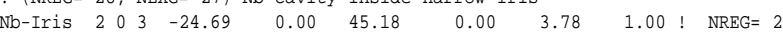

! (NREG $=29$, NEXG $=28$ ) Vacuum inside the cavity

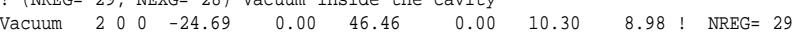

! (NREG $=30, \mathrm{NEXG}=29) \mathrm{Nb}$ cavity inside wide

$\begin{array}{llllllllll}\mathrm{Nb}-\text { cavi2 } & 2 & 0 & 3 & -24.69 & 0.00 & 46.18 & 0.00 & 10.58 & 9.54\end{array} \quad$ NREG $=30$

! $\quad(\mathrm{NREG}=31, \mathrm{NEXG}=30) \mathrm{Nb}$ cavity inside narrow Iris

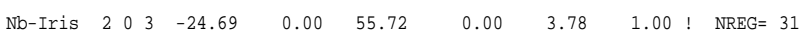

! (NREG $=32, \mathrm{NEXG}=31) \mathrm{Nb}$ cavity inside narrow Iris

Nb-Iris $\begin{array}{llllllllll}2 & 0 & 3 & -24.69 & 0.00 & 56.72 & 0.00 & 3.78 & 1.00 & !\end{array}$

! (NREG $=33, \mathrm{NEXG}=32$ ) Vacuum inside the cavity

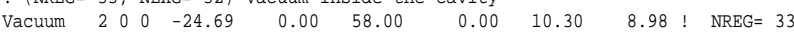

! $(\mathrm{NREG}=34, \mathrm{NEXG}=33) \mathrm{Nb}$ cavity inside wide

$\begin{array}{llllllllll}\mathrm{Nb}-\text { cavi2 } & 2 & 0 & 3 & -24.69 & 0.00 & 57.72 & 0.00 & 10.58 & 9.54\end{array} \quad$ NREG $=34$

! (NREG $=35, \mathrm{NEXG}=34) \mathrm{Nb}$ cavity inside narrow Iris

$\begin{array}{lllllllllll}\mathrm{Nb} \text {-Iris } & 2 & 0 & 3 & -24.69 & 0.00 & 67.26 & 0.00 & 3.78 & 1.00 & \text { ! }\end{array}$

! (NREG $=36, \quad$ NEXG $=35)$ Nb cavity inside narrow Iris

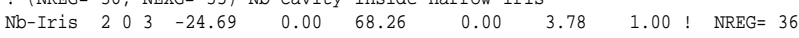

! (NREG $=37, \mathrm{NEXG}=36$ ) Vacuum inside the cavity

$\begin{array}{llllllllll}\text { Vacuum } & 2 & 0 & 0 & -24.69 & 0.00 & 69.54 & 0.00 & 10.30 & 8.98\end{array} \quad$ NREG $=37$

! (NREG= 38, NEXG $=37) \quad \mathrm{Nb}$ cavity inside wide

$\begin{array}{lllllllllll}\mathrm{Nb}-\text { cavi2 } & 2 & 0 & 3 & -24.69 & 0.00 & 69.26 & 0.00 & 10.58 & 9.54 & \text { NREG }=38\end{array}$

! $(\mathrm{NREG}=39, \mathrm{NEXG}=38) \mathrm{Nb}$ cavity inside narrow Iris

$\begin{array}{llllllllll}\mathrm{Nb}-\text { Iris } & 2 & 0 & 3 & -24.69 & 0.00 & 78.80 & 0.00 & 3.78 & 1.00\end{array} \quad$ NREG $=39$

! (NREG $=40, \mathrm{NEXG}=39$ ) $\mathrm{Nb}$ cavity inside narrow Iris

$\begin{array}{llllllllll}\mathrm{Nb} \text {-Iris } & 2 & 0 & 3 & -24.69 & 0.00 & 79.80 & 0.00 & 3.78 & 1.00\end{array} \quad$ NREG $=40$

I $(\mathrm{NREG}=41, \mathrm{NEXG}=40)$ Vacuum inside the cavity

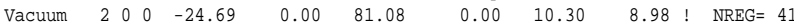

! (NREG $=42, \mathrm{NEXG}=41) \mathrm{Nb}$ cavity inside wide

$\begin{array}{llllllllll}\mathrm{Nb}-\mathrm{cavi} 2 & 2 & 0 & 3 & -24.69 & 0.00 & 80.80 & 0.00 & 10.58 & 9.54\end{array} \quad$ NREG $=42$

! (NREG $=43, \mathrm{NEXG}=42) \mathrm{Nb}$ cavity inside narrow Iris

$\mathrm{Nb}$-Iris $\begin{array}{lllllllll}2 & 0 & 3 & -24.69 & 0.00 & 90.34 & 0.00 & 3.78 & 1.00\end{array} \quad$ NREG $=43$

! (NREG $=44, \mathrm{NEXG}=43$ ) $\mathrm{Nb}$ cavity inside narrow Iris

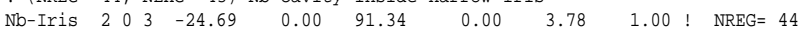

! $(\mathrm{NREG}=45, \mathrm{NEXG}=44)$ Vacuum inside the cavity

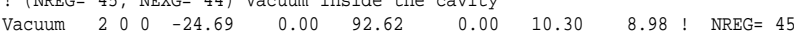

! (NREG $=46, \mathrm{NEXG}=45) \mathrm{Nb}$ cavity inside wide

$\begin{array}{lllllllllll}\mathrm{Nb}-\text { cavi2 } & 2 & 0 & 3 & -24.69 & 0.00 & 92.34 & 0.00 & 10.58 & 9.54 & \text { n NREG }=46\end{array}$

! (NREG $=47$, NEXG $=46)$ Nb cavity inside narrow Iris

$\begin{array}{llllllllll}\mathrm{Nb} \text {-Iris } & 2 & 0 & 3 & -24.69 & 0.00 & 101.88 & 0.00 & 3.78 & 1.00\end{array} \quad \mathrm{NREG}=47$

! (NREG $=48, \mathrm{NEXG}=47$ ) $\mathrm{Nb}$ cavity inside narrow Iris

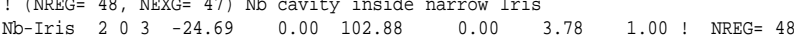

! (NREG $=49$, NEXG $=48$ ) Vacuum inside the cavity

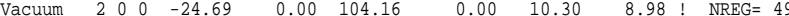

! (NREG $=50, N E X G=49) \mathrm{Nb}$ cavity inside wide

$\begin{array}{llllllllll}\mathrm{Nb}-\text { cavi2 } & 2 & 0 & 3 & -24.69 & 0.00 & 103.88 & 0.00 & 10.58 & 9.54\end{array} \quad \mathrm{NREG}=50$

! $(\mathrm{NREG}=51, \mathrm{NEXG}=50) \mathrm{Nb}$ cavity inside narrow Iris

Nb-Iris $\begin{array}{ccccccccc}2 & 0 & 3 & -24.69 & 0.00 & 113.42 & 0.00 & 3.78 & 1.00\end{array} \quad$ NREG $=51$

! (NREG $=52$, NEXG $=51$ ) HOM coupler

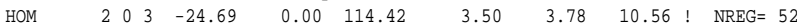

! (NREG $=53$, NEXG $=52$ ) gap between previous HOM and next HOM

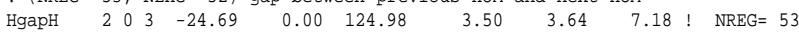

! (NREG $=54$, NEXG $=53$ ) LHE Liquid He

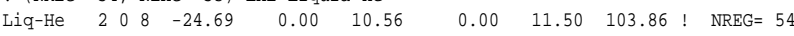

! (NREG= 55, NEXG $=54$ ) Ti outside cover of cavity

$\begin{array}{llllllllll}\text { Ti-cavi } & 2 & 0 & 4 & -24.69 & 0.00 & 10.06 & 0.00 & 12.00 & 104.86\end{array} \quad$ NREG $=55$

! (NREG $=56, \quad$ NEXG $=55$ ) mu metal outside cover of cavit

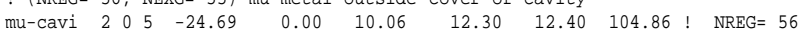

NREG $=15^{\sim} 56$ is repeated 8 times with different $z$-coordinate

! (NREG=350, NEXG=349) mu metal outside cover of cavity

$\begin{array}{llllllllll}\text { ! (NREG=350, } & \text { NEXG=349) } & \text { mu metal outside cover of cavity } & & \\ \text { mu-cavi } & 2 & 0 & 5 & -24.69 & 0.00 & 935.18 & 12.30 & 12.40 & 104.86 !\end{array}$

! (NREG=351, NEXG=350) Yellow Beamline equipment after 8 9-cell cavity

$\begin{array}{lllllllllll}\text { Yellow } & 2 & 0 & 2 & -24.69 & 0.00 & 1057.28 & 11.43 & 11.94 & 74.73 & \text { ! NREG }=351 \\ \text { STOP } & & & & & & & & & \end{array}$ 